УДК 622.692.23

ВЛИЯНИЕ УВЕЛИЧЕНИЯ ТОЛЩИНЫ СТЕНКИ РЕЗЕРВУАРА

НА СНИЖЕНИЕ ПОКАЗАТЕЛЯ РИСКА ИХ ЭКСПЛУАТАЦИИ

\title{
EFFECT OF TANK WALL THICKNESS INCREASING \\ ON OPERATION RISK REDUCTION
}

\author{
В.А. Самсонова, М.X. Хуснияров
}

Уфимский государственный нефтяной технический университет, г. Уфа, Российская федерация

\author{
Vera A. Samsonova, Mirat Kh. Khusniyarov \\ Ufa State Petroleum Technological University, \\ Ufa, Russian Federation \\ e-mail: my-vera-81@mail.ru
}

\begin{abstract}
Аннотация. Расположение резервуарных парков регламентируется требованиям нормативно-технической документации. Но спроектированные и построенные несколько десятилетий назад настоящие резервуарные парки уже не отвечают современным требованиям в области промышленной безопасности: многие из них расположены в непосредственной близости от промышленных и гражданских объектов, построенных позже. Для приведения объекта к требуемым нормам на эксплуатирующих предприятиях разрабатываются мероприятия, компенсирующие отступления от требований норм и правил в области промышленной безопасности. Одним из мероприятий по повышению безопасности эксплуатации резервуаров в резервуарных парках является увеличение расчетной толщины основных элементов конструкций резервуара (стенка, днище). В данной статье рассмотрены подходы к
\end{abstract}


оценке влияния увеличения толщины стенки на вероятность повреждения резервуара.

Abstract. The location of tank farms is regulated by the requirements of regulatory and technical documentation. But real tank farms, designed and built several decades ago, no longer meet modern requirements in the field of industrial safety: many of them are located in close proximity to industrial and civil facilities built later. To bring the object to the required standards at operating enterprises, measures are being developed to compensate for deviations from the requirements of standards and regulations in the field of industrial safety. One of the measures to improve the safety operation of tanks in tank farms is to increase the calculated thickness of the main structural elements of the tank (wall, bottom). This article discusses approaches to assessing the impact of increasing wall thickness on the probability of damage to the tank.

Ключевые слова: анализ риска, дерево отказов, резервуарный парк, физический износ, вероятность отказа, компенсирующие мероприятия, толщина стенки резервуара

Key words: risk analysis, fault tree, tank farm, physical deterioration, probability of failure, compensating actions, tank wall thickness

Вертикальные стальные резервуары широко используются на предприятиях нефтегазовой отрасли для хранения нефти, нефтепродуктов и других жидкостей, необходимых для реализации технологических процессов этих предприятий. Резервуары и резервуарные парки встречаются практически во всех крупных населенных пунктах нашей страны. Это связано с тем, что нефтепродукты являются основными энергоносителями и необходимы по всей территории страны. Расположение резервуарных парков по отношению к другим промышленным и гражданским объектам регламентируются 
нормативными документами (НТД) в области пожарной и промышленной безопасности. При проектировании и строительстве данных объектов требования НТД очень строго соблюдаются. В силу различных причин построенные с учетом всех требований НТД несколько десятилетий назад резервуарные парки в настоящее время оказались в непосредственной близи от других промышленных и гражданских объектов, которые были построены значительно позже и с несоблюдением нормативных расстояний до резервуарного парка. Данная ситуация является серьезной, так как данные нарушения не позволяют выполнять мероприятия по реконструкции и техническому перевооружению в резервуарных парках.

Парадоксальность ситуации заключается в том, что в соответствии с требованиями НТД для резервуарных парков необходимо внедрение современных систем противоаварийной автоматической защиты (ПАЗ), современных систем пожарной защиты и т.д., а выполнение работ по разработке проектной документации и внедрение этих систем запрещается этими же НТД в связи с несоблюдением нормативных расстояний до соседних объектов.

Учитывая создавшуюся ситуацию, законодательно утверждена процедура разработки и экспертизы промышленной безопасности обоснования безопасности опасного производственного объекта. В соответствии с п. 12 ФНП «Правила безопасности для опасных производственных объектов магистральных трубопроводов»: «При отсутствии установленных требований по безопасным расстояниям или невозможности их соблюдения эти требования должны быть определены в обосновании безопасности опасного производственного объекта» [1]. Для приведения объекта к требуемым нормам на эксплуатирующих предприятия разрабатываются мероприятия, компенсирующие отступления от требования ФНП и правил в области промышленной безопасности. 
Одним из мероприятий по повышению безопасности эксплуатации резервуаров в резервуарных парках является увеличение расчетной толщины основных элементов конструкций РВС (стенка, днище). В данной статье рассмотрены подходы к оценке влияния увеличения толщины стенки на вероятность повреждения резервуара.

При проведении оценки риска аварии и определения значений приемлемого риска необходимо оценить вероятность разрушения основного технологического оборудования, к числу которых относятся резервуары. Для определения вероятности разрушения резервуара при разработке документации в области промышленной безопасности было построено дерево отказов (рисунок 1). Рядом с исходными событиями на дереве отказов указана вероятность данного события. При проведении мероприятий, компенсирующих отступления от требования ФНП и правил в области промышленной безопасности, вероятности некоторых исходных событий изменятся в меньшую сторону, что приведет к уменьшению вероятности конечного события и, тем самым, снижению риска.

С момента начала эксплуатации любое технологическое оборудование подвергается износу, который нарастает с увеличением срока эксплуатации объектов и приводит к потере ими части своей полезности под действием различных факторов устаревания и природно-временного воздействия.

Применительно к резервуарам хранения под физическим износом понимается ухудшение первоначальных технико-экономических свойств, обусловленное естественным изнашиванием рассматриваемого объекта в процессе эксплуатации и под воздействием различных природных факторов. Другими словами, это износ материалов, из которых создан резервуар, потеря его первоначальных качеств, постепенное разрушение конструкций и т.д. 


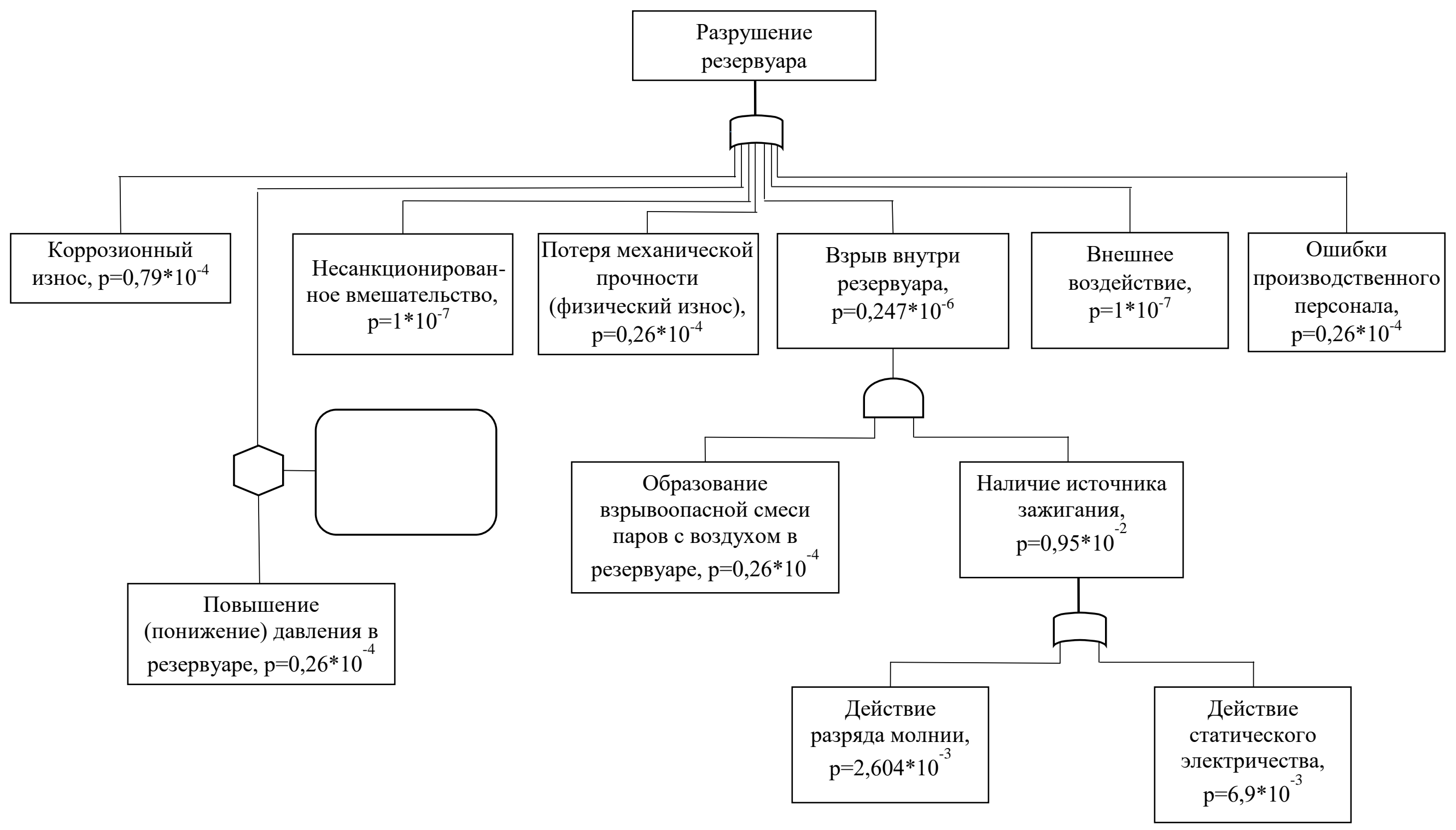

Рисунок 1. Дерево отказов 
Физический износ представляет собой естественный процесс ухудшения характеристик оборудования во время его эксплуатации под воздействием многих факторов, таких как: трение, коррозия, старение материалов, вибрация, флуктуации температуры и влажности, качество обслуживания и др. Рост физического износа приводит к увеличению вероятности аварийных отказов оборудования.

Исходя из определения понятия «физический износ» можно говорить о том, что данное событие зависит от проектируемого срока эксплуатации резервуара.

На рассматриваемом дереве отказов исходное событие «потеря механической прочности» «физический износ» имеет вероятность $0,26 * 10^{-4}$ [2]. Данная вероятность события соответствует резервуару, проектируемому для срока эксплуатации 20 лет ( $\left.T_{\mathrm{m} 1}\right)$ (Резервуар 1) [3]. При замене действующего резервуара на новый с увеличенной толщиной стенки срок эксплуатации естественно будет увеличиваться. Для резервуара с увеличением толщины стенки на 1 мм срок эксплуатации достигает 29 лет $\left(T_{\mathrm{m} 2}\right)$ (Резервуар 2). Изменение вероятности события предполагается спрогнозировать с помощью известных законов теории надежности [4].

Согласно [4], экспоненциальным распределением хорошо описывается случай, когда вероятность отказа не зависит от длительности предыдущего использования изделия, т.е. когда возникают в основном внезапные отказы.

$$
\begin{array}{r}
P(t)=e^{-\lambda t}, \\
\lambda=\frac{1}{T_{m}},
\end{array}
$$

следовательно:

$$
P(t)=e^{-\frac{t}{T_{m}}}
$$

где $\lambda$ - интенсивность отказов;

$t$ - время; 
$T_{\mathrm{m}}-$ средняя наработка до отказа.

При $t=T_{\mathrm{m}}$ получается $\lambda=1$ и

$$
P(t)=e^{-1}=0,37 .
$$

Графическая зависимость изменения вероятности безотказной работы для двух рассматриваемых резервуаров приведена на рисунке 2. Прямая соответствует вероятности безотказной работы 0,37.

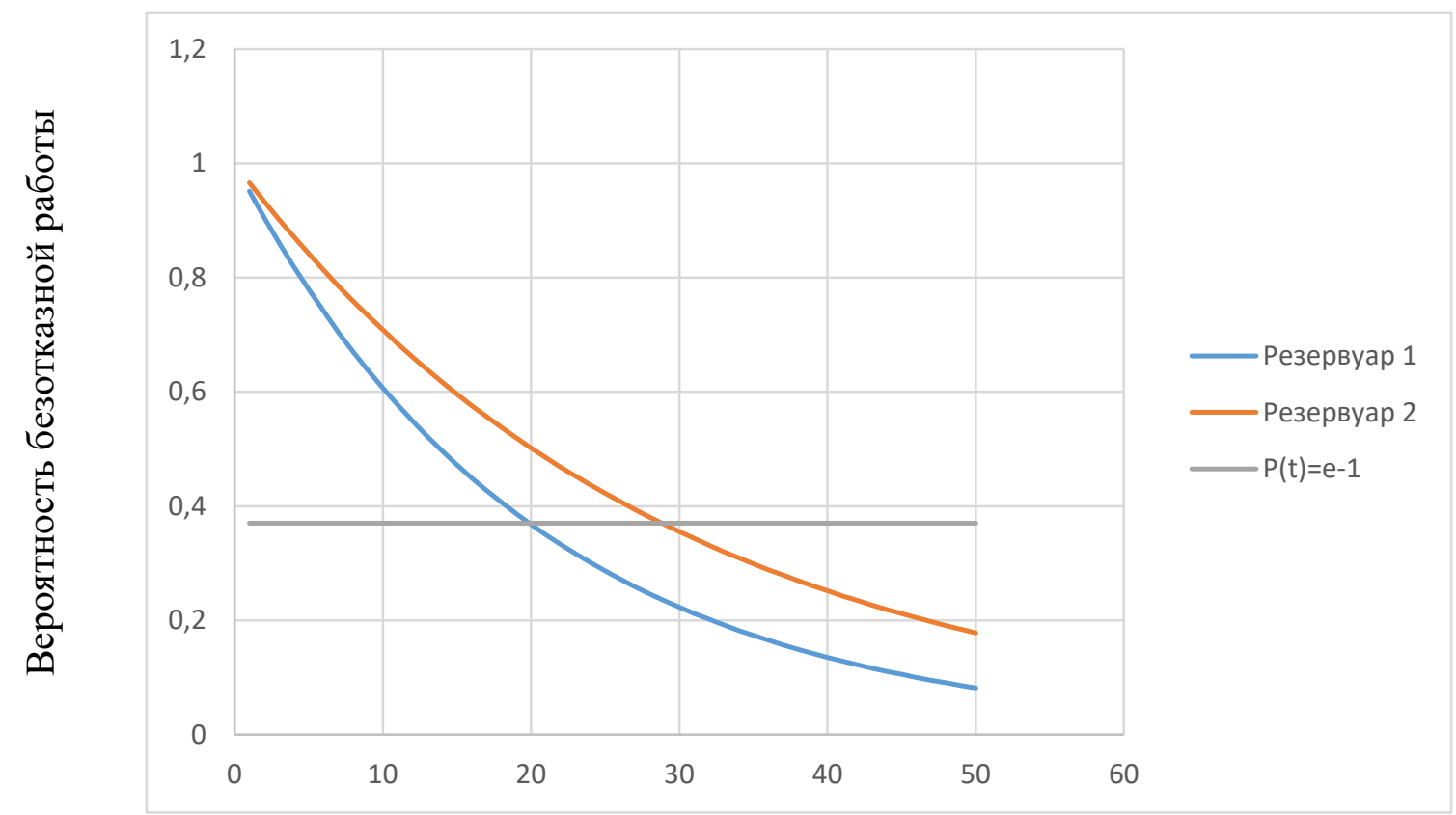

Время, годы

Рисунок 2. Изменение вероятности безотказной работы от времени

$P(t)=0,37$ означает, что при внезапных отказах после работы одинаковых изделий в течение $t=T_{\mathrm{m}}, 63 \%$ из них откажут, а $37 \%$ останутся работоспособными. Для современного оборудования, применяемого на опасных производственных объектах, требуется высокая вероятность безотказной работы - от $P(t)=0,99$ до $P(t)=0,9999$ и выше. Для значений $P(t)>0,9$ формула (1) с достаточной для практики точностью представляется в следующем виде:

$$
P(t)=1-\lambda t=1-\frac{t}{T_{m}} .
$$


Данная формула получена при линейной аппроксимации участка кривой $P(t)$ для значений, близких единице:

$$
P(t)+Q(t)=1 .
$$

Используя уравнения (5) и (6), получено:

$$
\begin{array}{r}
1-Q(t)=1-\frac{t}{T_{m}}, \\
Q(t)=\frac{t}{T_{m}} .
\end{array}
$$

Внезапный отказ для выбранного оборудования происходит в произвольный момент времени. При рассмотрении Резервуара 1 и Резервуара 2 возможно предположить, что $t_{1}=t_{2}$. Исходя из этого следует:

$$
\frac{Q_{1}(t)}{T_{m 1}}=\frac{Q_{2}(t)}{T_{m 2}} .
$$

Зная значения времени наработки до отказа и вероятности отказа Резервуара 1, можно вычислить значения вероятности отказа Резервуара 2:

$$
Q_{2}(t)=0,18 * 10^{-4} .
$$

Так же можно это проиллюстрировать графически (рисунок 3).

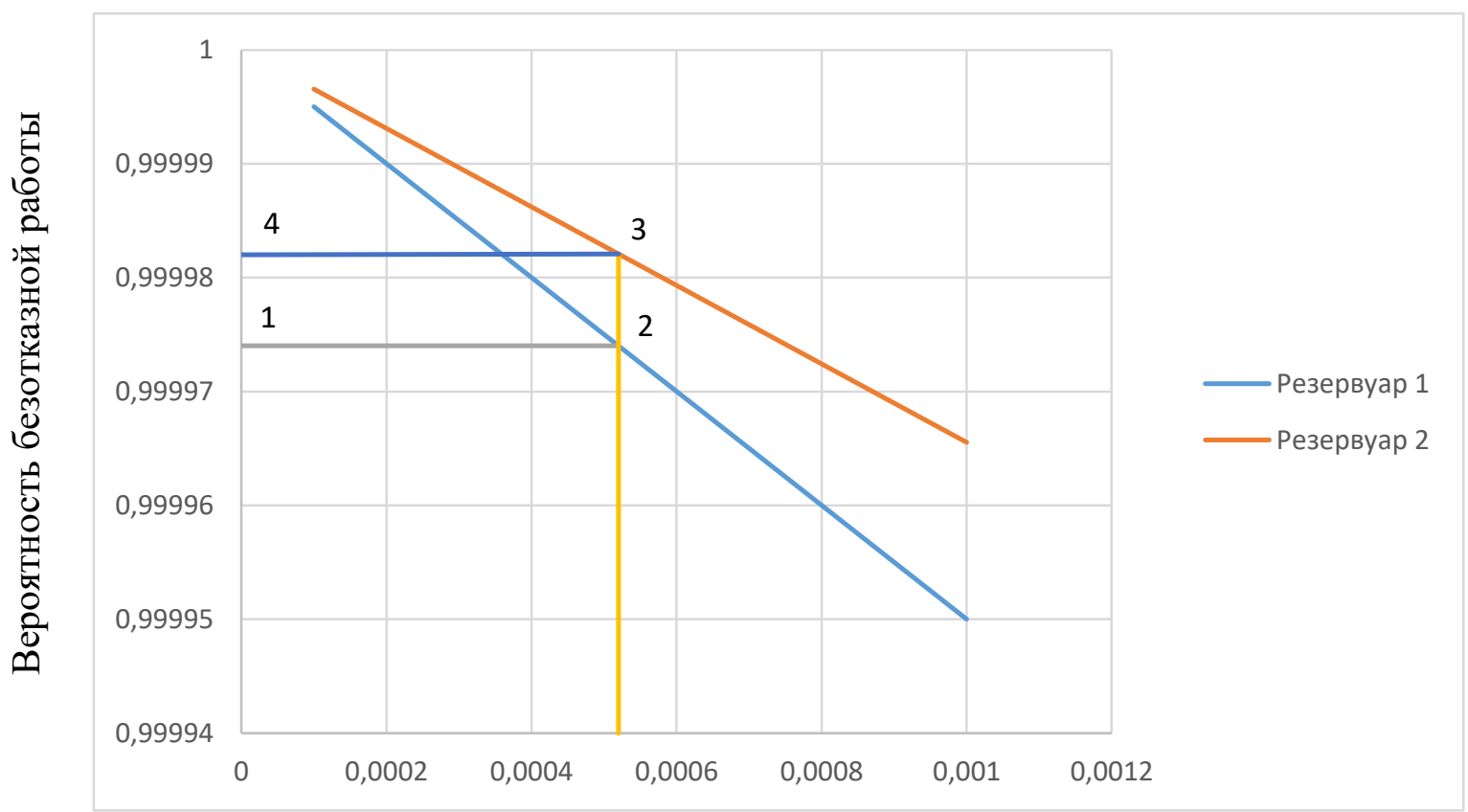

Время, годы

Рисунок 3. Зависимости вероятности безотказной работы для Резервуара 1 и Резервуара 2 
На графике представлены зависимости $P(t)$ для Резервуара 1 и Резервуара 2. Через точку 1 , соответствующую вероятности безотказной работы $P_{1}(t)=1-Q_{1}(t)=1-0,000026=0,999974$, проводится прямая, параллельная оси абсцисс, до пересечения с кривой вероятности безотказной работы Резервуара 1 (точка 2). Проведя прямую через точку 2 , параллельную оси ординат, до пересечения с кривой вероятности безотказной работы резервуара 2 получаем точку 3. Через полученную точку3, параллельно оси абсцисс, проводится прямая до пересечения с осью ординат (точка 4). Полученная точка 4 на оси ординат соответствует значению вероятности безотказной работы Резервуара 2 в тот же момент времени.

Значение вероятности безотказной работы, соответствующее точке 4 , равно 0,999982. Тогда вероятность отказа:

$$
Q_{1}(t)=1-P_{1}(t)=1-0,999982=0,000018 .
$$

Таким образом, при замене Резервуара 1 со сроком службы 20 лет на Резервуар 2 со сроком службы 29 лет вероятность отказа снизится до $0,18 * 10^{-4}$. Вероятность разрушения резервуара при изменении вероятности исходного события «Потеря механической прочности (физический износ)» снизится с $1,314 * 10^{-4}$ до $1,234 * 10^{-4}$.

\section{Вывод}

Исходя из полученного меньшего значения вероятности разрушения резервуара проведение компенсирующего мероприятия, направленного на увеличение расчетной толщины основных элементов конструкций РВС (стенка, днище) на 1 мм, оправданно и позволяет снизить значения риска. 


\section{Список используемых источников}

1. Федеральные нормы и правила в области промышленной безопасности «Правила безопасности для опасных производственных объектов магистральных трубопроводов». М.: ЗАО НТЦ ПБ, 2014.

2. Анализ риска и проблем безопасности: в 4 ч. М.: МГФ «Знание», 2007. Ч. 3: Прикладные вопросы анализа рисков критически важных объектов / Научный руководитель К.В. Фролов. 815 с.

3. ГОСТ 31385-2016. Резервуары вертикальные цилиндрические стальные для нефти и нефтепродуктов. Общие технические условия. М.: Стандартинформ, 2016.

4. Проников А.С. Параметрическая надежность машин. М.: Изд-во МГТУ им. Баумана, 2002. 560 с.

\section{References}

1. Federal'nye normy $i$ pravila $v$ oblasti promyshlennoi bezopasnosti «Pravila bezopasnosti dlya opasnykh proizvodstvennykh ob"ektov magistral'nykh truboprovodov»»[Safety Regulations for Hazardous Production Facilities of Trunk Pipelines]. Moscow, ZAO STC PB Publ., 2014. [in Russian].

2. Analiz riska i problem bezopasnosti: v 4 ch. [Risk and Safety Analysis: in 4 ch.]. Moscow, MGF «Znanie», 2007. Ch. 3: Prikladnye voprosy analiza riskov kriticheski vazhnykh ob"ektov (Nauchnyi rukovoditel' K.V. Frolov) [Ch. 3: Applied Risk Analysis of Critical Facilities]. 815 p. [in Russian].

3. GOST 31385-2016. Rezervuary vertikal'nye tsilindricheskie stal'nye dlya nefti i nefteproduktov. Obshchie tekhnicheskie usloviya [State Standard 313852016. Vertical Cylindrical Steel Tanks for Petroleum and Petroleum Products. General Specifications]. Moscow, STANDARTINFORM, 2016. [in Russian].

4. Pronikov A.S. Parametricheskaya nadezhnost' mashin [Parametric Machine Reliability]. Moscow, Bauman MSTU Publ., 2002. 560 p. [in Russian]. 


\section{Сведения об авторах}

\section{About the authors}

Самсонова Вера Александровна, канд. техн. наук, доцент кафедры «Газохимия и моделирование химико-технологических процессов», УГНТУ, г. Уфа, Российская Федерация

Vera A. Samsonova, Candidate of Engineering Sciences, Assistant Professor of Gas Chemistry and Modeling of Chemical Processes Department, USPTU, Ufa, Russian Federation

e-mail: my-vera-81@mail.ru

Хуснияров Мират Ханифович, д-р техн. наук, профессор, профессор кафедры «Автоматизация технологических процессов и производств», УГНТУ, г. Уфа, Российская Федерация

Mirat Kh. Khusniyarov, Doctor of Engineering Sciences, Associated Professor, Professor of Automation of Technological Processes and Production Department, USPTU, Ufa, Russian Federation

e-mail: xmirat@mail.ru 